\title{
Stable Standing Waves of Nonlinear Klein-Gordon Equations
}

\section{Jalal Shatah}

Division of Applied Mathematics, Brown University, Providence, RI 02912, USA

\begin{abstract}
In this paper we give sufficient conditions for the stability of the standing waves of least energy for nonlinear Klein-Gordon equations.
\end{abstract}

\section{Introduction}

In this paper we give sufficient conditions for the stability of standing waves of the nonlinear Klein-Gordon equation:

$$
u_{t t}-\Delta u+u+f(|u|) \arg u=0, \quad x \in \mathbb{R}^{n}, \quad n>2,
$$

or equivalently the steady-state solutions of the modulated equation:

$$
u_{t t}+2 i \omega u_{t}-\Delta u+\left(1-\omega^{2}\right) u+f(|u|) \arg u=0 .
$$

We show the stability of the standing waves of lowest energy in the energy norm. They are stable with respect to the lowest energy solution set of

$$
-\Delta u+\left(1-\omega^{2}\right) u+f(|u|) \arg u=0 \text {. }
$$

The existence of solutions of $(0.3 \omega)$ has already been shown in [9] and [10]. In the generality presented in Sect. I this problem was solved by Berestycki and Lions in [10]. The condition for stability is very simple. If we define

$$
d(\omega)=1 / 2 \int\left|\nabla \varphi_{\omega}\right|^{2} d x+\left(1-\omega^{2}\right) / 2 \int\left|\varphi_{\omega}\right|^{2} d x+\int G\left(\left|\varphi_{\omega}\right|\right) d x,
$$

where $G^{\prime}=f$ and $\varphi_{\omega}$ is a least energy solution of $(0.3 \omega)$, then:

Theorem. If $d(\omega)$ is strictly convex in a neighborhood of $\omega_{0}$, then $\varphi_{\omega_{0}}$ is stable.

Equation (0.1) arises in particle physics. It models the field equation for spin- 0 particles [4]. The existence of stable standing waves has, until now, eluded any rigorous proof. Anderson [1] showed by numerical computation that these equations can have stable standing waves. He studied the particular example 
where $f(|u|) \arg u=-|u|^{2} u+|u|^{4} u, x \in \mathbb{R}^{3}$, and showed numerically that there are both stable and unstable standing waves. We have shown in [6] the existence of unstable standing waves for this example when $\omega$ is close to 1 . Here we show that $d(\omega)$ is strictly convex for some $\omega$ and therefore there are stable standing waves. This problem was subsequently considered by Lee [4] and others who arrive at the same conclusion, heuristically, using the principle of least energy.

It can be shown that the condition $d(\omega)$ is convex, is equivalent to the condition that the energy of Eq. $(0.1) E(u, v)$ restricted to the charge $Q(u, v)=Q\left(\varphi_{\omega}, i \omega \varphi_{\omega}\right)$ has a local minimum at $\left(\varphi_{\omega}, i \omega \varphi_{\omega}\right)$, where the charge $Q(u, v) \equiv \operatorname{Im} \int u \bar{v} d x$. This agrees with the physical intuition of the problem [4].

The theory of linearized stability does not give a clue to whether there are stable standing waves or not. The spectrum of the linearized problem might lie entirely on the imaginary axis and therefore one cannot deduce the stability of these waves.

It is interesting to compare this result of stability with the instability result of the ground state, i.e., the least energy steady state solution of Eq. (0.1). Berestycki and Cazenave [2] showed that for special type of nonlinearities, solutions that are close to the ground state blow up in finite time. In [6] we generalized this result to show instability, but not necessarily blow up, of the ground state for all nonlinearities that we can prove the existence of a ground state for.

Finally, for the Schrödinger equation: $i u_{t}-\Delta u+f(|u|) \arg u=0$. Cazenave and Lions [3] showed the existence of stable standing waves for some nonlinearities. Berestycki and Cazenave [2] showed the existence of unstable standing waves for another type of nonlinearities.

Notation. We employ here the standard notation

$$
\begin{gathered}
H_{r}^{1}\left(\mathbb{R}^{n}\right)=\left\{u, \text { radially symmetric functions on } \mathbb{R}^{n}\right. \\
\left.\|u\| \equiv\left(\int|\nabla u(x)|^{2} d x+\int|u(x)|^{2} d x\right)^{1 / 2}<\infty\right\}, \\
L_{r}^{p}\left(\mathbb{R}^{n}\right)=\left\{u, \text { radially symmetric function on } \mathbb{R}^{n}\right. \\
\left.|u|_{p}=\left(\int|u(x)|^{p} d x\right)^{1 / p}<\infty\right\},
\end{gathered}
$$

$C_{0 r}^{\infty}\left(\mathbb{R}^{n}\right)=\{$ radially symmetric, infinitely differentiable functions

with compact support\},

$$
\begin{gathered}
f(s)=o(s) \Leftrightarrow|f(s) / s| \rightarrow 0 \text { as }|s| \rightarrow 0, \\
f(s)=O(s) \Leftrightarrow|f(s) / s| \text { is bounded as } s \rightarrow 0 .
\end{gathered}
$$

\section{Standing Waves}

Consider the nonlinear Klein-Gordon equation

$$
u_{t t}-\Delta u+u+f(|u|) \arg u=0, \quad f(0)=f^{\prime}(0)=0, \quad x \in \mathbb{R}^{n}, \quad n>2 .
$$

This equation has nontrivial standing waves, $u(x, t)=e^{i \omega t} \varphi(x)$ provided that

$$
-\Delta \varphi+\left(1-\omega^{2}\right) \varphi+f(|\varphi|) \arg \varphi=0
$$


has a nontrivial solution.

\section{Definition 1.1. Let}

$$
J_{\omega}(\psi)=1 / 2 \int|\nabla \psi|^{2} d x+\left(1-\omega^{2}\right) / 2 \int|\psi|^{2} d x+\int G(|\psi|) d x
$$

where $G^{\prime}(|\psi|)=f(|\psi|)$ and $G(0)=0$,

$$
\begin{aligned}
K_{\omega}(\psi) & \equiv(n-2) / 2 \int|\nabla \psi|^{2} d x+n\left(\left(1-\omega^{2}\right) / 2 \int|\psi|^{2} d x+\int G(|\psi| d x),\right. \\
M_{\omega} & \equiv\left\{\psi \in H_{r}^{1}\left(\mathbb{R}^{n}\right), K_{\omega}(\psi)=0, \psi \neq 0\right\} .
\end{aligned}
$$

In order that Eq. $(0.3 \omega)$ has nontrivial solutions it is sufficient that $f$ and $G$ satisfy [10]:

$$
H \begin{cases}H .1 & \exists \eta>0 \ni: G(\eta)<0 \\ H .2 & \lim _{\eta \rightarrow \infty} f(\eta) / \eta^{l} \geqq 0, \quad l<1+4 /(n-2) .\end{cases}
$$

Definition 1.2. Let $\omega^{*}=\left\{\inf \omega \geqq 0 \ni: \exists \eta,\left(1-\omega^{2}\right) \eta^{2} / 2+G(\eta)<0\right\}$. Thus $\omega^{*} \in[0,1)$. We shall always take $\omega^{*}<\omega<1$.

Lemma 1.1. For $\omega \in\left(\omega^{*}, 1\right) M_{\omega}$ is a $C^{1}$ hypersurface in $H_{r}^{1}\left(\mathbb{R}^{n}\right)$ bounded away from zero.

Proof. See [6].

Proposition 1.1. If $\varphi_{\omega} \in H_{r}^{1}\left(\mathbb{R}^{n}\right)$ is a solution of $(0.3 \omega)$, and $\int G\left(\left|\varphi_{\omega}\right|\right) d x<\infty$, then

$$
K_{\omega}\left(\varphi_{\omega}\right)=0 \text {. }
$$

Proof. Let $\varphi_{\beta}(x)=\varphi_{\omega}(x / \beta)$, then

$$
J_{\omega}\left(\varphi_{\beta}\right)=\beta^{n-2} / 2 \int\left|\nabla \varphi_{\omega}\right|^{2} d x+\beta^{n}\left(\left(1-\omega^{2}\right) / 2 \int\left|\varphi_{\omega}\right|^{2} d x+\int G\left(\left|\varphi_{\omega}\right|\right) d x\right),
$$

since $\varphi_{\omega}$ is a solution then $\delta J_{\omega}\left(\varphi_{\omega}\right)=0 \Rightarrow d\left(J_{\omega}\left(\varphi_{\beta}\right)\right) /\left.d \beta\right|_{\beta=1}=0$, but

$$
d\left(J_{\omega}\left(\varphi_{\beta}\right)\right) /\left.d \beta\right|_{\beta=1}=(n-2) / 2 \int\left|\nabla \varphi_{\omega}\right|^{2} d x+n\left(1-\omega^{2}\right) / 2 \int\left|\varphi_{\omega}\right|^{2} d x+\int G\left(\left|\varphi_{\omega}\right| d x\right),
$$

therefore $K_{\omega}\left(\varphi_{\omega}\right)=0$.

Theorem 1.1. Let $\omega^{2} \in\left(\omega^{* 2}, 1\right), n>2$, then

$$
d(\omega) \equiv \inf _{v \in M_{\omega}} J_{\omega}(v)
$$

is achieved for some $v \neq 0$, and

$$
d(\omega)=\left\{\inf 1 / n \int|\nabla v|^{2} d x, K_{\omega}(v) \leqq 0, v \neq 0\right\} .
$$

Moreover $v$ satisfies

$$
-\Delta v+\left(1-\omega^{2}\right) v+f(|v|) \arg v=0 .
$$

Proof. First we show the equivalence of both minimization problems. Consider any function $v \in H_{r}^{1}\left(\mathbb{R}^{n}\right)$ such that $K_{\omega}(v)<0$. Let $v_{\beta}(x)=v(x / \beta)$. Then

$$
K_{\omega}\left(v_{\beta}\right)=\beta^{n-2}(n-2) / 2 \int|\nabla v|^{2} d x+\beta^{n} n\left(\left(1-\omega^{2}\right) / 2 \int|v|^{2} d x+\int G(|v|) d x\right) .
$$


Now for $\beta=1 K_{\omega}\left(v_{1}\right)=K_{\omega}(v)<0$ and for $\beta$ close to zero $K_{\omega}\left(v_{\beta}\right)>0$. Therefore there exist a $\beta_{0} \in(0,1)$ such that $K_{\omega}\left(v_{\beta_{0}}\right)=0$, and

$$
1 / n \int\left|\nabla v_{\beta_{0}}\right|^{2} d x=\beta_{0}^{n-2} / n \int|\nabla v|^{2} d x<1 / n \int|\nabla v| 2 d x .
$$

Since $J_{\omega}(v)=1 / n\left(\int|\nabla v|^{2} d x+K_{\omega}(v)\right)$, then

$$
\begin{aligned}
d(\omega) & \equiv \inf _{v \in M_{\omega}} J_{\omega}(v)=\inf \left\{1 / n \int|\nabla v|^{2} d x, K_{\omega}(v)=0, v \neq 0\right\} \\
& =\inf \left\{1 / n \int|\nabla v|^{2} d x, K_{\omega}(v) \leqq 0, v \neq 0\right\} .
\end{aligned}
$$

Next, consider any minimizing sequence $v_{k}$. Then $\left(\int\left|\nabla v_{k}\right|^{2} d x\right)$ is bounded. By H.2 for every $\varepsilon>0$ there exist $C_{1}(\varepsilon)>0$ such that $G(\eta)>-\varepsilon / 2 \eta^{2}-C_{1}(\varepsilon) \eta^{l+1}$, where $l<1$ $+4 /(n-2)$. Since $K_{\omega}\left(v_{k}\right) \leqq 0$, then

$$
0 \geqq K_{\omega}\left(v_{k}\right)=(n-2) / 2 \int\left|\nabla v_{k}\right|^{2} d x+n\left(\left(1-\omega^{2}\right) / 2 \int\left|v_{k}\right|^{2} d x+\int G\left(\left|v_{k}\right|\right) d x\right)
$$

and this implies

$$
0 \geqq(n-2) / 2 \int\left|\nabla v_{k}\right|^{2} d x+n\left(\left(1-\omega^{2}-\varepsilon\right) / 2 \int\left|v_{k}\right|^{2} d x-C_{1}(\varepsilon) \int\left|v_{k}\right|^{l+1} d x\right) .
$$

Now by Sobolev embedding $H_{r}^{1}\left(\mathbb{R}^{n}\right) \rightarrow L_{r}^{p}\left(\mathbb{R}^{n}\right), 2<p<2+4 /(n-2)$ and since $\left(\int\left|\nabla v_{k}\right|^{2} d x\right)$ is bounded we get that $\left\|v_{k}\right\|$ is bounded. Therefore there exist a subsequence, also denote it by $\left(v_{k}\right)$, such that

$$
v_{k} \stackrel{w}{=} v_{0} \in H_{r}^{1}\left(\mathbb{R}^{n}\right) \quad \text { and } \quad v_{k} \rightarrow v_{0} \in L^{p} \quad 2<p<2+4 /(n-2),
$$

since for radially symmetric $H_{r}^{1}\left(\mathbb{R}^{n}\right) \hookrightarrow L_{r}^{p}\left(\mathbb{R}^{n}\right)$ is compact for $2<p<2+4 /(n-2)$. By lower semicontinuity of weak limits we have:

$$
\begin{aligned}
K_{\omega}\left(v_{0}\right) & =(n-2) / 2 \int\left|\nabla v_{0}\right|^{2} d x+n\left(\left(1-\omega^{2}\right) / 2 \int\left|v_{0}\right|^{2} d x+\int G\left(\left|v_{0}\right|\right) d x\right) \\
& \leqq \lim _{k \rightarrow \infty}(n-2) / 2 \int\left|\nabla v_{k}\right|^{2} d x+n\left(\left(1-\omega^{2}\right) / 2 \int\left|v_{k}\right|^{2} d x+\int G\left(\left|v_{k}\right|\right) d x\right)=0
\end{aligned}
$$

And from the above argument the inequalities are equalities and the weak limit is strong. Consequently $v_{0} \neq 0$ by Lemma 1.1 , and

$$
d(\omega)=\inf _{v \in M_{\omega}} J_{\omega}(v)=J_{\omega}\left(v_{0}\right)
$$

Finally, to show that $v_{0}$ satisfies Eq. (0.3) we have by the Lagrange multiplier method

$$
\delta J_{\omega}\left(v_{0}\right)=\lambda \delta K_{\omega}\left(v_{0}\right)
$$

or

$$
\begin{aligned}
- & \Delta v_{0}+\left(1-\omega^{2}\right) v_{0}+f\left(\left|v_{0}\right|\right) \arg v_{0} \\
& =\lambda\left(-(n-2) \Delta v_{0}+n\left(1-\omega^{2}\right) v_{0}+n f\left(\left|v_{0}\right|\right) \arg v_{0}\right) .
\end{aligned}
$$

By Proposition 1.1 we have

$$
\begin{aligned}
& (n-2) / 2 \int\left|\nabla v_{0}\right|^{2} d x+n\left(\left(1-\omega^{2}\right) / 2 \int\left|v_{0}\right|^{2} d x+\int G\left(\left|v_{0}\right|\right) d x\right) \\
& \quad=\lambda\left[(n-2)^{2} / 2 \int\left|\nabla v_{0}\right|^{2} d x+n^{2}\left(\left(1-\omega^{2}\right) / 2 \int\left|v_{0}\right|^{2} d x+\int G\left(\left|v_{0}\right|\right) d x\right)\right] .
\end{aligned}
$$


But

$$
K_{\omega}\left(v_{0}\right)=(n-2) / 2 \int\left|\nabla v_{0}\right|^{2} d x+n\left(\left(1-\omega^{2}\right) / 2 \int\left|v_{0}\right|^{2} d x+\int G\left(\left|v_{0}\right|\right) d x\right)=0,
$$

therefore

$$
\begin{aligned}
& 0=\lambda\left[(n-2)^{2} / 2 \int\left|\nabla v_{0}\right|^{2} d x-n(n-2) / 2 \int\left|\nabla v_{0}\right|^{2} d x\right], \\
& 0=\lambda(n-2) \int\left|\nabla v_{0}\right|^{2} d x,
\end{aligned}
$$

and this implies that $\lambda=0$.

Definition 1.3. Let $S_{\omega}$ be the solution set of $d(\omega)=\inf _{v \in M_{\omega}} J_{\omega}(v)$.

Corollary 1.1. $S_{\omega}$ is also the solution set of

$$
\inf J_{\omega}(v)=d(\omega), \quad \omega^{2} \in\left(\omega^{* 2}, 1\right), \quad 1 / n \int|\nabla v|^{2} d x=d(\omega) .
$$

Proof. Suppose $\exists v$ such that $1 / n \int|\nabla v|^{2} d x=d(\omega)$ and $J_{\omega}(v)<d(\omega)$. Then

$$
1 / n K_{\omega}(v)=J_{\omega}(v)-1 / n \int|\nabla v|^{2} d x<0 .
$$

But by Theorem 1.1

$$
d(\omega)=\inf \left\{1 / n \int|\nabla v|^{2} d x, K_{\omega}(v) \leqq 0, v \neq 0\right\},
$$

and this contradicts the above assumption. Therefore

$$
\inf J_{\omega}(v)=d(\omega), \quad 1 / n \int|\nabla v|^{2} d x=d(\omega) .
$$

Now to show that the solution set of this problem is $S_{\omega}$ we note that $\forall v$ which is a minimum we have

$$
\delta J_{\omega}(v)=\lambda \Delta v, \quad \text { or } \quad-(1+\lambda) \Delta v+\left(1-\omega^{2}\right) v+f(|v|) \arg v=0,
$$

and by Proposition 1.1

$$
\begin{aligned}
& (1+\lambda)(n-2) / 2 \int|\nabla v|^{2} d x+n\left(\left(1-\omega^{2}\right) / 2 \int|v|^{2} d x+\int G(|v| d x)=0\right. \\
& \quad \Rightarrow J_{\omega}(v)=1 / 2 \int|\nabla v|^{2} d x-(1+\lambda)(n-2) /(2 n) \int|\nabla v|^{2} d x=d(\omega) \\
& \quad \Rightarrow J_{\omega}(v)=1 / n \int|\nabla v|^{2} d x-\lambda(n-2) /(2 n) \int|\nabla v|^{2} d x=d(\omega) \\
& \Rightarrow \lambda=0 \text { and } \therefore K_{\omega}(v)=0 \Rightarrow v \in S_{\omega} .
\end{aligned}
$$

Corollary 1.2. Let $v^{k} \in H_{r}^{1}\left(\mathbb{R}^{n}\right)$ be a sequence such that $1 / n \int\left|\nabla v^{k}\right|^{2} d x \rightarrow d(\omega)$ and $J_{\omega}\left(v^{k}\right) \rightarrow d^{1} \leqq d(\omega)$, then $v^{k}$ has a strongly convergent subsequence $v^{k} \rightarrow \varphi_{\omega} \in H_{r}^{1}\left(\mathbb{R}^{n}\right)$ for some $\varphi_{\omega} \in S_{\omega}$ and

$$
\left|\int\left(G\left(\left|v^{k}\right|\right)-G\left(\left|\varphi_{\omega}\right|\right)\right) d x\right| \rightarrow 0, \quad d^{1}=d(\omega) .
$$

Proof. Since $\int\left|\nabla v^{k}\right|^{2} d x$ and $J_{\omega}\left(v^{k}\right)$ are bounded, $v^{k}$ is a bounded sequence in $H_{r}^{1}\left(\mathbb{R}^{n}\right)$ (see the proof of Theorem 1.1). $v^{k}$ has a weakly convergent subsequence, also denote it by $v^{k}$, such that

$$
v^{k} \stackrel{w}{\rightarrow} v_{0} \in H_{r}^{1}\left(\mathbb{R}^{n}\right), \quad v^{k} \rightarrow v_{0} \in L_{r}^{p}, \quad 2<p<2+4 /(n-2) .
$$

Now $1 /\left.n|| \nabla v_{0}\right|^{2} d x \leqq \lim _{k \rightarrow \infty} 1 / n \int\left|\nabla v^{k}\right|^{2} d x=d(\omega)$, and $K_{\omega}\left(v_{0}\right) \leqq \lim _{k \rightarrow \infty} K_{\omega}\left(v^{k}\right)$ (by the proof 
of Theorem 1.1), therefore

$$
K_{\omega}\left(v_{0}\right) \leqq n \varliminf_{k \rightarrow \infty}\left(J_{\omega}\left(v^{k}\right)-1 / n \int\left|\nabla v^{k}\right|^{2} d x\right)
$$

or

$$
K_{\omega}\left(v_{0}\right) \leqq d^{1}-d(\omega) \leqq 0 .
$$

But from Theorem 1.1 we have

$$
d(\omega)=\inf \left\{1 / n \int|\nabla v|^{2} d x, K_{\omega}(v) \leqq 0, v \neq 0\right\} .
$$

Therefore all inequalities are equalities and the weak convergence is strong. Therefore

$$
\int G\left(\left|v^{k}\right|\right) d x \rightarrow \int G\left(\left|\varphi_{\omega}\right|\right) d x \quad \text { and } \quad d^{1}=d(\omega) .
$$

Since $1 / n \int\left|\nabla v_{0}\right|^{2} d x=d(\omega)$ and $K_{\omega}\left(v_{0}\right)=0 \Rightarrow v_{0} \in S_{\omega}$ and $\therefore v^{k} \rightarrow v_{0} \in H_{r}^{1}\left(\mathbb{R}^{n}\right), v_{0} \in S_{\omega}$.

Remark 1.1. This is the only place where radial symmetry is needed. One can generalize the above result to include the space $H^{1}\left(\mathbb{R}^{n}\right)$ by using the notion of "concentrated compactness" introduced by Lions [5]. In this case the sequence $v_{k} \in H^{1}\left(\mathbb{R}^{n}\right)$ of Corollary 1.2 will have a subsequence $v_{k_{n}}$ such that $v_{k_{n}}\left(\cdot+y_{k_{n}}\right) \in H^{1}\left(\mathbb{R}^{n}\right)$ is relatively compact in $H^{1}\left(\mathbb{R}^{n}\right)$ for some sequence $\left(y_{k_{n}}\right)$.

\section{Standing Waves as a Function of Frequency}

In this section we'll study the behavior of $d(\omega)=1 / n \int\left|\nabla \varphi_{\omega}\right|^{2} d x$ as a function of the frequency $\omega$.

Lemma 2.1. Let $\omega_{1}<\omega_{2}$ be such that $\left[\omega_{1}, \omega_{2}\right] \subset\left(\omega^{*}, 1\right)$, then $d(\omega)$ and $\int\left|\varphi_{\omega}\right|^{2} d x\left(\varphi_{\omega} \in S_{\omega}\right)$ are uniformly bounded in $\omega \in\left[\omega_{1}, \omega_{2}\right]$.

Proof. Since $K$ is continuous in $\omega, d(\omega)$ is bounded for $\omega \in\left[\omega_{1}, \omega_{2}\right]$. Now for $\varphi \in S_{\omega}$, $K_{\omega}\left(\varphi_{\omega}\right)=0$. By $H .2 G(\eta) \geqq-c \eta^{l+1} ; l<1+4 /(n-2)$, for $\eta$ large, and $G(0)=G^{\prime}(0)$ $=G^{\prime \prime}(0)=0 \Rightarrow$ for any $a>0, \frac{a}{2} \eta^{2}+G(\eta)>-C_{a} \eta^{l_{0}+1}, l_{0}=1+4 /(n-2)$,

$$
\int\left(\frac{a}{2}|v|^{2}+G(|v|)\right) d x \geqq-C_{a} \int|v|^{l_{0}+1} d x .
$$

Now because $K_{\omega}\left(\varphi_{\omega}\right)=0$, and by Sobolev embedding

$$
(n-2) / 2 \int\left|\nabla \varphi_{\omega}\right|^{2} d x+n\left(1-\omega^{2}-a^{2}\right) / 2 \int\left|\varphi_{\omega}\right|^{2} d x-C_{a}\left(\int\left|\nabla \varphi_{\omega}\right|^{2} d x\right)^{\alpha} \leqq 0
$$

for a small.

This implies that $\int\left|\varphi_{\omega}\right|^{2} d x$ is uniformly bounded for $\omega \in\left[\omega_{1}, \omega_{2}\right] \subset\left(\omega^{*}, 1\right)$.

Proposition 2.1. a) $d(\omega)$ is a decreasing function of $\omega \in\left(\omega^{*}, 1\right)$, b) if $\omega_{1}<\omega_{2}$,

$$
\begin{aligned}
& d\left(\omega_{2}\right)<d\left(\omega_{1}\right)-\left(\omega_{2}^{2}-\omega_{1}^{2}\right) / 2 \int\left|\varphi_{\omega_{1}}\right|^{2} d x+o\left(\omega_{1}-\omega_{2}\right) \\
& d\left(\omega_{1}\right)<d\left(\omega_{2}\right)+\left(\omega_{2}^{2}-\omega_{1}^{2}\right) / 2 \int\left|\varphi_{\omega_{2}}\right|^{2} d x+o\left(\omega_{1}-\omega_{2}\right) .
\end{aligned}
$$

Consequently, $d(\omega)$ is a continuous function of $\omega \in\left(\omega^{*}, 1\right)$. 
Proof. a) Let $\omega_{1}<\omega_{2}$, then

$$
K_{\omega_{2}}\left(\varphi_{\omega_{1}}\right)=(n-2) / 2 \int\left|\nabla \varphi_{\omega_{1}}\right|^{2} d x+n\left(\left(1-\omega_{2}^{2}\right) / 2 \int\left|\varphi_{\omega_{1}}\right|^{2} d x+\int G\left(\left|\varphi_{\omega_{1}}\right|\right) d x\right)
$$

or

$$
\begin{gathered}
K_{\omega_{2}}\left(\varphi_{\omega_{1}}\right)=(n-2) / 2 \int\left|\nabla \varphi_{\omega_{1}}\right|^{2} d x+n\left(\left(1-\omega_{1}^{2}\right) / 2 \int\left|\varphi_{\omega_{1}}\right|^{2} d x\right. \\
\left.+G\left(\left|\varphi_{\omega_{1}}\right|\right) d x\right)-n\left(\omega_{2}^{2}-\omega_{1}^{2}\right) / 2 \int\left|\varphi_{\omega_{1}}\right| d x, \\
K_{\omega_{2}}\left(\varphi_{\omega_{1}}\right)=K_{\omega_{1}}\left(\varphi_{\omega_{1}}\right)-n\left(\omega_{2}^{2}-\omega_{1}^{2}\right) / 2 \int\left|\varphi_{\omega_{1}}\right|^{2} d x,
\end{gathered}
$$

but $K_{\omega_{1}}\left(\varphi_{\omega_{1}}\right)=0$

$$
\Rightarrow K_{\omega_{2}}\left(\varphi_{\omega_{1}}\right)=-n\left(\omega_{2}^{2}-\omega_{1}^{2}\right) / 2 \int\left|\varphi_{\omega_{1}}\right|^{2} d x<0 .
$$

Therefore

$$
d\left(\omega_{1}\right)=1 / n \int\left|\nabla \varphi_{\omega_{1}}\right|^{2} d x>\inf \left\{1 / n \int|\nabla v|^{2} d x, K_{\omega_{2}}(v) \leqq 0, v \neq 0\right\},
$$

since $K_{\omega_{2}}\left(\varphi_{\omega_{1}}\right)<0$,

$$
\Rightarrow d\left(\omega_{1}\right)>d\left(\omega_{2}\right) .
$$

b) Again let $\omega_{1}<\omega_{2}$ and $\psi_{\beta}(x)=\varphi_{\omega_{1}}(x / \beta)$, then

$$
\begin{gathered}
K_{\omega_{2}}\left(\psi_{\beta}\right)=(n-2) \beta^{n-2} / 2 \int\left|\nabla \varphi_{\omega_{1}}\right|^{2} d x+n \beta^{n}\left(\left(1-\omega_{2}^{2}\right) / 2 \int\left|\varphi_{\omega_{1}}\right|^{2} d x+\int G\left(\left|\varphi_{\omega_{1}}\right|\right) d x\right), \\
K_{\omega_{2}}\left(\psi_{\beta}\right)=(n-2) \beta^{n-2} / 2 \int\left|\nabla \varphi_{\omega_{1}}\right|^{2} d x-\beta^{n}\left((n-2) / 2 \int\left|\nabla \varphi_{\omega_{1}}\right|^{2} d x\right. \\
\left.+n\left(\omega_{2}^{2}-\omega_{1}^{2}\right) / 2 \int\left|\varphi_{\omega_{1}}\right|^{2} d x\right) .
\end{gathered}
$$

Let

$$
\Delta_{12}=\left(\omega_{2}^{2}-\omega_{1}^{2}\right) / 2 \int\left|\varphi_{\omega_{1}}\right|^{2} d x,
$$

then $K_{\omega_{2}}\left(\psi_{\beta}\right)=\left(n(n-2) d\left(\omega_{1}\right) / 2\right) \beta^{n-2}-n\left((n-2) d\left(\omega_{1}\right) / 2+\Delta_{12}\right) \beta^{n}$, and for

$$
\begin{gathered}
\beta_{2} \equiv 1 /\left(1+2 \Delta_{12} /(n-2) d\left(\omega_{1}\right)\right)^{1 / 2}, \quad K_{\omega_{2}}\left(\psi_{\beta_{2}}\right)=0, \\
\therefore d\left(\omega_{2}\right) \leqq 1 / n \int\left|\nabla \psi_{\beta_{2}}\right|^{2} d x=\beta_{2}^{n-2} / n \int\left|\nabla \varphi_{\omega_{1}}\right|^{2} d x=\beta_{2}^{n-2} d\left(\omega_{1}\right) .
\end{gathered}
$$

But for $\omega_{1}-\omega_{2}$ small, $\left|\Delta_{12}\right|<C\left(\omega_{2}^{2}-\omega_{1}^{2}\right)$, since $\int\left|\varphi_{\omega}\right|^{2} d x$ is bounded. Therefore

$$
\beta_{2}^{n-2}=1-\Delta_{12} / d\left(\omega_{1}\right)+o\left(\Delta_{12}\right)
$$

and from Eq. (2.3) we get

$$
d\left(\omega_{2}\right) \leqq d\left(\omega_{1}\right)-\Delta_{12}+o\left(\Delta_{12}\right),
$$

or

$$
d\left(\omega_{2}\right) \leqq d\left(\omega_{1}\right)-\left(\omega_{2}^{2}-\omega_{1}^{2}\right) / 2 \int\left|\varphi_{\omega_{1}}\right|^{2} d x+o\left(\omega_{2}-\omega_{1}\right) .
$$

To show the second part of b) let $\psi_{\gamma}(x)=\varphi_{\omega_{2}}(x / \gamma)$, then

$$
K_{\omega_{1}}\left(\psi_{\gamma}\right)=(n-2) \gamma^{n-2} / 2 \int\left|\nabla \varphi_{\omega_{2}}\right|^{2} d x+n \gamma^{n}\left(\left(1-\omega_{1}^{2}\right) / 2 \int\left|\varphi_{\omega_{2}}\right|^{2} d x+\int G\left(\left|\varphi_{\omega_{2}}\right|\right) d x\right),
$$

or

$$
K_{\omega_{1}}\left(\psi_{\gamma}\right)=\left(n(n-2) d\left(\omega_{2}\right) / 2\right) \gamma^{n-2}-n\left((n-2) d\left(\omega_{2}\right) / 2-\Delta_{21}\right) \gamma^{n},
$$


where

$$
\Delta_{21}=\left(\omega_{2}^{2}-\omega_{1}^{2}\right) / 2 \int\left|\varphi_{\omega_{2}}\right|^{2} d x
$$

since $\int\left|\nabla \varphi_{\omega_{2}}\right|^{2} d x=n d\left(\omega_{2}\right)$ and $K_{\omega_{2}}\left(\varphi_{\omega_{2}}\right)=0$.

For

$$
\begin{gathered}
\gamma_{1} \equiv 1 /\left(1-2 \Delta_{21} /(n-2) d\left(\omega_{2}\right)\right)^{1 / 2}, \\
K_{\omega_{1}}\left(\psi_{\gamma_{1}}\right)=0 \Rightarrow d\left(\omega_{1}\right) \leqq 1 / n \int\left|\nabla \psi_{\gamma_{1}}\right|^{2} d x=\gamma_{1}^{n-2} / n \int\left|\nabla \varphi_{\omega_{2}}\right|^{2} d x=\gamma_{1}^{n-2} d\left(\omega_{2}\right),
\end{gathered}
$$

but for $\omega_{1}-\omega_{2}$ small

$$
\begin{gathered}
\gamma_{1}^{n-2}=1+\Delta_{21} / d\left(\omega_{2}\right)+o\left(\omega_{2}-\omega_{1}\right), \\
\therefore d\left(\omega_{1}\right)<d\left(\omega_{2}\right)+\left(\omega_{2}^{2}-\omega_{1}^{2}\right) / 2 \int\left|\varphi_{\omega_{2}}\right|^{2} d x+o\left(\omega_{1}-\omega_{2}\right) .
\end{gathered}
$$

The continuity of $d(\omega)$ follows from Eq. (2.6) and (2.11).

Next we'll need this lemma about strictly convex functions.

Lemma 2.2. Suppose $h(\omega)$ is a strictly convex function in a neighborhood of $\omega_{0}$, then $\forall \varepsilon>0 \quad \exists N(\varepsilon)>0 \ni:\left|\omega_{\varepsilon}-\omega_{0}\right|=\varepsilon$,

a) $-\omega_{\varepsilon}<\omega_{0}<\omega,\left|\omega-\omega_{0}\right|<\varepsilon / 2$

$$
\left(h\left(\omega_{\varepsilon}\right)-h(\omega)\right) /\left(\omega_{\varepsilon}-\omega\right) \leqq\left(h\left(\omega_{0}\right)-h(\omega)\right) /\left(\omega_{0}-\omega\right)-1 / N(\varepsilon),
$$

b) $\omega<\omega_{0}<\omega_{\varepsilon},\left|\omega-\omega_{0}\right|<\varepsilon / 2$

$$
\left(h\left(\omega_{\varepsilon}\right)-h(\omega)\right) /\left(\omega_{\varepsilon}-\omega\right) \geqq\left(h\left(\omega_{0}\right)-h(\omega)\right) /\left(\omega_{0}-\omega\right)+1 / N(\varepsilon) .
$$

Proof. The proof is very easy to see geometrically from the picture below.
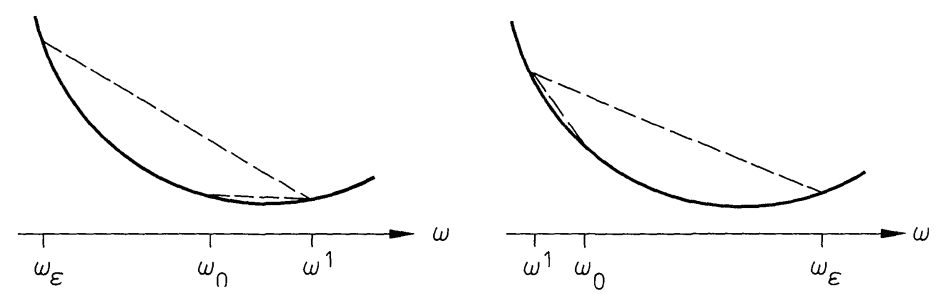

Fig. 2.1

We'll give a proof for the case $\omega_{\varepsilon}<\omega_{0}<\omega$ and the second part follows by an identical argument. Assume that the claim is false. Then there is an $\varepsilon_{0}>0$ and a sequence $\omega_{k} \ni:\left|\omega_{\varepsilon_{0}}-\omega_{0}\right|=\varepsilon_{0},\left|\omega_{k}-\omega_{0}\right|<\varepsilon / 2$,

$$
\left(h\left(\omega_{0}\right)-h\left(\omega_{k}\right)\right) /\left(\omega_{0}-\omega_{k}\right)-1 / k<\left(h\left(\omega_{\varepsilon_{0}}\right)-h\left(\omega_{k}\right)\right) /\left(\omega_{\varepsilon_{0}}-\omega_{k}\right) .
$$

Pick $\omega_{1}$ such that $\omega_{\varepsilon_{0}}<\omega_{1}<\omega_{0}$, then

$$
\left(h\left(\omega_{0}\right)-h\left(\omega_{k}\right)\right) /\left(\omega_{0}-\omega_{k}\right)>\left(h\left(\omega_{1}\right)-h\left(\omega_{k}\right)\right) /\left(\omega_{1}-\omega_{k}\right)
$$

[since $h(\omega)$ is convex]. From Eq. (2.12) we get

$$
\left(h\left(\omega_{\varepsilon_{0}}\right)-h\left(\omega_{k}\right)\right) /\left(\omega_{\varepsilon_{0}}-\omega_{k}\right)>\left(h\left(\omega_{1}\right)-h\left(\omega_{k}\right)\right) /\left(\omega_{1}-\omega_{k}\right)-1 / k .
$$


Since $\omega_{k}$ is bounded it has a convergent subsequence. Also denote it by $\omega_{k}$ such that $\omega_{k} \rightarrow \omega_{2} \geqq \omega_{0}>\omega_{1}>\omega_{\varepsilon_{0}}$. Now from Eq. (2.14) and continuity of $h(\omega)$

$$
\left(h\left(\omega_{\varepsilon_{0}}\right)-\dot{h}\left(\omega_{2}\right)\right) /\left(\omega_{\varepsilon_{0}}-\omega_{2}\right) \geqq\left(h\left(\omega_{1}\right)-h\left(\omega_{2}\right)\right) /\left(\omega_{1}-\omega_{2}\right) .
$$

But since $h(\omega)$ is strictly convex

$$
\left(h\left(\omega_{1}\right)-h\left(\omega_{2}\right)\right) /\left(\omega_{1}-\omega_{2}\right)>\left(h\left(\omega_{\varepsilon_{0}}\right)-h\left(\omega_{2}\right)\right) /\left(\omega_{\varepsilon_{0}}-\omega_{2}\right),
$$

which contradicts Eq. (2.15). Therefore the claim is true.

Theorem 2.1. Suppose that $d(\omega)$ is strictly convex in a neighborhood of $\omega_{0}$, then for $\omega$ close to $\omega_{0} \exists \eta(\omega)>0, \eta\left(\omega_{0}\right)=0$, such that

$$
d(\omega)-d\left(\omega_{0}\right) \geqq\left(\omega_{0}-\omega\right) \omega_{0} \int\left|\varphi_{\omega_{0}}\right|^{2} d x+\eta(\omega) .
$$

Proof. Let $\omega<\omega_{0}, \omega$ close to $\omega_{0}$. Then from Lemma 2.2 and for $\omega<\omega_{0}<\omega_{1}$,

$$
\left(d\left(\omega_{1}\right)-d(\omega)\right) /\left(\omega_{1}-\omega\right) \leqq\left(d\left(\omega_{1}\right)-d\left(\omega_{0}\right)\right) /\left(\omega_{1}-\omega_{0}\right)-1 / N(\omega),
$$

and from Proposition 2.1

$$
\left(d\left(\omega_{1}\right)-d\left(\omega_{0}\right)\right) /\left(\omega_{1}-\omega_{0}\right)<-\left(\omega_{1}+\omega_{0}\right) / 2 \int\left|\varphi_{\omega_{0}}\right|^{2} d x+o\left(\omega_{1}-\omega_{0}\right) /\left(\omega_{1}-\omega_{0}\right) .
$$

From Eq. (2.17) and (2.18)

$$
\left(d\left(\omega_{1}\right)-d(\omega)\right) /\left(\omega_{1}-\omega\right) \leqq-\left(\omega_{1}+\omega_{0}\right) / 2 \int\left|\varphi_{\omega_{0}}\right|^{2} d x-1 / N(\omega)+o\left(\omega_{1}-\omega_{0}\right) /\left(\omega_{1}-\omega_{0}\right)
$$

Let $\omega_{1} \rightarrow \omega_{0}$, then by continuity of $d(\omega)$

$$
\left(d\left(\omega_{0}\right)-d(\omega)\right) /\left(\omega_{0}-\omega\right) \leqq-\omega_{0} \int\left|\varphi_{\omega_{0}}\right|^{2} d x-1 / N(\omega)
$$

or

$$
d(\omega)-d\left(\omega_{0}\right) \geqq \omega_{0}\left(\omega_{0}-\omega\right) \int\left|\varphi_{\omega_{0}}\right|^{2} d x+\left(\omega_{0}-\omega\right) / N(\omega) .
$$

For $\omega>\omega_{0}$, from Lemma 2.2 and $\omega>\omega_{0}>\omega_{1}$, we have

$$
\left(d(\omega)-d\left(\omega_{1}\right)\right) /\left(\omega-\omega_{1}\right) \geqq\left(d\left(\omega_{0}\right)-d\left(\omega_{1}\right)\right) /\left(\omega_{0}-\omega_{1}\right)+1 / N(\omega),
$$

and from Proposition 2.1

$$
\left(d\left(\omega_{0}\right)-d\left(\omega_{1}\right)\right) /\left(\omega_{0}-\omega_{1}\right) \geqq\left(\omega_{0}+\omega_{1}\right) / 2 \int\left|\varphi_{\omega_{0}}\right|^{2} d x+o\left(\omega_{1}-\omega_{0}\right) /\left(\omega_{1}-\omega_{0}\right) .
$$

Again from Eq. (2.20) and (2.21) and letting $\omega_{1} \rightarrow \omega_{0}$,

$$
\left(d(\omega)-d\left(\omega_{0}\right)\right) \geqq-\omega_{0}\left(\omega-\omega_{0}\right) \int\left|\varphi_{\omega_{0}}\right|^{2} d x+\left(\omega-\omega_{0}\right) / N(\omega),
$$

and this concludes the proof of Theorem 2.1.

\section{Stability of the Standing Waves}

Now if we consider the Cauchy problem

$$
\begin{gathered}
u_{t t}-\Delta u+u+f(|u|) \arg u=0, \quad x \in \mathbb{R}^{n}, \\
u(0)=u_{0} \in H_{r}^{1}\left(\mathbb{R}^{n}\right), \quad u_{t}(0)=u_{1} \in L_{r}^{2}\left(\mathbb{R}^{n}\right),
\end{gathered}
$$


we don't have strong solutions $\left(u(\cdot) \in C\left([0, T], H_{r}^{1}\left(\mathbb{R}^{n}\right)\right), u_{t}(\cdot) \in C\left([0, T), L_{r}^{2}\left(\mathbb{R}^{n}\right)\right)\right)$ for the general nonlinearities we are considering; but we always have weak solutions

$$
\left(u(\cdot) \in L^{\infty}\left([0, T), H_{r}^{1}\left(\mathbb{R}^{n}\right)\right), u_{t}(\cdot) \in L^{\infty}\left([0, T), L_{r}^{2}\left(\mathbb{R}^{n}\right)\right)\right),
$$

that are weakly continuous in $t$. Also we don't necessarily have uniqueness, or energy identity, but we always can find a weak solution that satisfies the energy inequality

$$
1 / 2 \int\left|u_{t}(t)\right|^{2} d x+J_{0}\left(u(t) \leqq 1 / 2 \int\left|u_{1}\right|^{2} d x+J_{0}\left(u_{0}\right)\right.
$$

provided $\int G\left(\left|u_{0}\right|\right) d x<\infty$ (see Strauss [8]).

Definition 3.1. Define the metric space $X=\left\{\right.$ completion of $u \in C_{0 r}^{\infty}\left(\mathbb{R}^{n}\right)$ with the metric

$$
\varrho\left(u_{1}, u_{2}\right)=\left\|u_{1}-u_{2}\right\|+\left|\int\left(G\left(\left|u_{1}\right|\right)-G\left(\left|u_{2}\right|\right)\right) d x\right|
$$

and define the modulated energy functional of Eq. $(0.2 \omega), E_{\omega}(u, v)=1 / 2 \int|v|^{2} d x$ $+J_{\omega}(u), u \in X, v \in L_{r}^{2}\left(\mathbb{R}^{n}\right)$.

$$
\begin{aligned}
R_{\omega}^{1} & \equiv\left\{u \in X, v \in L_{r}^{2}\left(\mathbb{R}^{n}\right) ; E_{\omega}(u, v)<d(\omega), K_{\omega}(u)>0\right\} \cup\{(0,0)\} \\
& =\left\{u \in X, v \in L_{r}^{2}\left(\mathbb{R}^{n}\right) ; E_{\omega}(u, v)<d(\omega), 1 / n \int|\nabla u|^{2} d x<d(\omega)\right\}, \\
R_{\omega}^{2} & \equiv\left\{u \in X, v \in L_{r}^{2}\left(\mathbb{R}^{n}\right) ; E_{\omega}(u, v)<d(\omega), K_{\omega}(u)<0, u \neq 0\right\} \\
& =\left\{u \in X, v \in L_{r}^{2}\left(\mathbb{R}^{n}\right) ; E_{\omega}(u, v)<d(\omega), 1 / n \int|\nabla u|^{2} d x>d(\omega)\right\} .
\end{aligned}
$$

Lemma 3.1. $R_{\omega}^{1}$ and $R_{\omega}^{2}$ are invariant regions under the flow of $(0.2 \omega)$ for the solutions that satisfy the energy inequality.

Proof. We'll prove this by contradiction. Let $\left(u_{0}, u_{1}\right) \in R_{\omega}^{1}$ and assume that there exist a $t_{1}$ such that $\left(u\left(t_{1}\right), u_{t}\left(t_{1}\right)\right) \notin R_{\omega}^{1}$. By lower semi-continuity of $K_{\omega}(u(t))$ there exist a minimal $t_{0}$ such that $\left(u\left(t_{0}\right), u_{t}\left(t_{0}\right)\right) \notin R_{\omega}^{1}$, i.e. $K_{\omega}\left(u\left(t_{0}\right)\right) \leqq 0$ and $K_{\omega}(u(t))>0$ for $t \in\left[0, t_{0}\right)$. Now

$$
\begin{aligned}
1 / n \int\left|\nabla u\left(t_{0}\right)\right|^{2} d x & \leqq \varliminf_{\substack{t \rightarrow t_{0} \\
t<t_{0}}} 1 / n \int|\nabla u(t)|^{2} d x \\
& \leqq \varliminf_{\substack{t \rightarrow t_{0} \\
t<t_{0}}} 1 / n\left(\int|\nabla u(t)|^{2} d x+K_{\omega}(u(t)),\right.
\end{aligned}
$$

therefore

$$
1 / n \int\left|\nabla u\left(t_{0}\right)\right|^{2} d x \leqq \varliminf_{t \rightarrow t_{0}} J_{\omega}(u(t)) \leqq \lim _{t \rightarrow t_{0}} E_{\omega}\left(u(t), u_{t}(t)\right)<d(\omega),
$$

and we also have $K\left(u\left(t_{0}\right)\right) \leqq 0$. This contradicts the definition of

$$
d(\omega)=\inf \left\{1 / n \int|\nabla v|^{2} d x, K_{\omega}(v) \leqq 0, v \neq 0\right\} .
$$

Therefore $R_{\omega}^{1}$ is invariant under the flow of Eq. $(0.2 \omega)$. Similarly we can show that $R_{\omega}^{2}$ is also invariant. 
Lemma 3.2. Let $u(t)$ be a solution of

$$
\begin{gathered}
u_{t t}-\Delta u+u+f(|u|) \arg u=0, \\
u(0)=u_{0} \in X, \quad u_{t}(0)=u_{1} \in L_{r}^{2}\left(\mathbb{R}^{n}\right),
\end{gathered}
$$

that satisfies the energy inequality. Then for every $K>0$ there exist $\delta(K)$ such that if

$$
\varrho\left(u_{0}, \varphi_{\omega_{0}}\right)+\left|u_{1}-i \omega_{0} \varphi_{\omega_{0}}\right|_{2}<\dot{\delta(K)},
$$

then $d\left(\omega_{0}+1 / K\right) \leqq 1 / n \int|\nabla u(t)|^{2} d x \leqq d\left(\omega_{0}-1 / K\right) \quad \forall t$.

Proof. Fix $K>0$ and let $\omega_{+}=\omega_{0}+1 / K, \omega_{-}=\omega_{0}-1 / K$, and $u(t)=v_{+}(t) e^{i \omega_{+} t}$ $=v_{-}(t) e^{i \omega-t}$. Then

$$
\begin{gathered}
v_{ \pm t t}+2 i \omega_{ \pm} v_{ \pm t}-\Delta v_{ \pm}+\left(1-\omega_{ \pm}^{2}\right) v_{ \pm}+f\left(\left|v_{ \pm}\right|\right) \arg v_{ \pm}=0 \\
v_{ \pm}(0)=u_{0}, \quad v_{ \pm t}(0)=u_{1}-i \omega_{ \pm} u_{0} .
\end{gathered}
$$

The energy inequality of this equation is

$$
\begin{aligned}
E_{\omega_{ \pm}}\left(v_{ \pm}(t), v_{ \pm t}(t)\right) \leqq & 1 / 2 \int\left|u_{1}-i \omega_{ \pm} u_{0}\right|^{2} d x+1 / 2 \int\left|\nabla u_{0}\right|^{2} d x \\
& +\left(1-\omega_{ \pm}^{2}\right) / 2 \int\left|u_{0}\right|^{2} d x+\int G\left(\left|u_{0}\right|\right) d x
\end{aligned}
$$

or

$$
1 / 2 \int\left|v_{ \pm t}(t)\right|^{2} d x+J_{\omega_{ \pm}}(u(t)) \leqq 1 / 2 \int\left|u_{1}-i \omega_{ \pm} u_{0}\right|^{2} d x+J_{\omega_{ \pm}}\left(u_{0}\right)
$$

but

$$
\begin{aligned}
\left|u_{1}-i \omega_{ \pm} u_{0}\right|_{2} \leqq & \left|u_{1}-i \omega_{0} \varphi_{\omega_{0}}\right|_{2}+\left|\omega_{0} \varphi_{\omega_{0}}-\omega_{ \pm} \varphi_{\omega_{0}}\right|_{2}+\left|\omega_{ \pm} \varphi_{\omega_{0}}-\omega_{ \pm} u_{0}\right|_{2} \\
& \Rightarrow 1 / 2 \int\left|u_{1}-i \omega_{ \pm} u_{0}\right|^{2} d x \leqq\left(\omega_{0}-\omega_{ \pm}\right)^{2} / 2 \int\left|\varphi_{\omega_{0}}\right|^{2} d x \\
& +C\left(\omega_{ \pm}, \omega_{0}\right) \int\left|u_{1}-i \omega_{0} \varphi_{\omega_{0}}\right|^{2} d x+\int\left|u_{0}-\varphi_{\omega_{0}}\right|^{2} d x
\end{aligned}
$$

Now since $d\left(\omega_{+}\right)<d\left(\omega_{0}\right)<d\left(\omega_{-}\right)$and

$$
d\left(\omega_{0}\right) \equiv 1 / n \int\left|\nabla \varphi_{\omega_{0}}\right|^{2} d x=1 / n \int\left|\nabla u_{0}\right|^{2} d x+O(\delta) .
$$

If we pick $\delta$ small enough we have

$$
\begin{aligned}
d\left(\omega_{+}\right)<1 / n \int\left|\nabla u_{0}\right|^{2} d x<d\left(\omega_{-}\right), \\
E_{\dot{\omega}_{ \pm}}\left(u_{0}, u_{1}-i \omega_{ \pm} u_{0}\right) \leqq\left(\omega_{0}-\omega_{ \pm}\right)^{2} / 2 \int\left|\varphi_{\omega_{0}}\right|^{2} d x+J_{\omega_{ \pm}}\left(\varphi_{\omega_{0}}\right)+O(\delta) \\
\leqq\left(\omega_{0}-\omega_{ \pm}\right) \omega_{0} \int\left|\varphi_{\omega_{0}}\right|^{2} d x+J_{\omega_{0}}\left(\varphi_{\omega_{0}}\right)+O(\delta),
\end{aligned}
$$

since $\left(\omega_{0}^{2}-\omega_{ \pm}^{2}\right) / 2+\left(\omega_{0}-\omega_{ \pm}\right)^{2} / 2=\left(\omega_{0}-\omega_{ \pm}\right) \omega_{0}$.

By Theorem 2.1 and for $\delta$ small

$$
\begin{aligned}
& \left(\omega_{0}-\omega_{ \pm}\right) \omega_{0} \int\left|\varphi_{\omega_{0}}\right|^{2} d x+d\left(\omega_{0}\right)+O(\delta) \\
& \quad \leqq\left(\omega_{0}-\omega_{ \pm}\right) \omega_{0} \int\left|\varphi_{\omega_{0}}\right|^{2} d x+d\left(\omega_{0}\right)+\eta\left(\omega_{ \pm}\right) \leqq d\left(\omega_{ \pm}\right),
\end{aligned}
$$

and therefore from Eq. (3.6) we have the energy inequality

$$
\begin{aligned}
& 1 / 2 \int\left|v_{ \pm t}(t)\right|^{2} d x+J_{\omega_{ \pm}}(u(t))<d\left(\omega_{ \pm}\right) \quad \forall t \\
& \quad \Rightarrow d\left(\omega_{ \pm}\right)<1 / n \int|\nabla u(t)|^{2} d x<d\left(\omega_{-}\right) \quad \forall t
\end{aligned}
$$

by Lemma 3.1. 
Theorem 3.1. If $d(\omega)$ is strictly convex at $\omega_{0}$, then the standing waves of frequency $\omega_{0}$ are stable in the following sense: for every $\varepsilon>0$ there exists a $\delta(\varepsilon)>0$ such that if $\varrho\left(u_{0}, \varphi_{\omega_{0}}\right)+\left|u_{1}-i \omega_{0} \varphi_{\omega_{0}}\right|_{2}<\delta(\varepsilon)$, then

$$
\inf _{\psi \in S_{\omega_{0}}}\left(\varrho(u(t), \psi)+\left|u_{t}(t)-i \omega_{0} \psi\right|_{2}\right)<\varepsilon \text { for all } t .
$$

Proof. Assume not. Then $\exists$ sequence $\left(u_{0_{k}}, u_{1_{k}}\right),\left(t^{k}\right)$ and an $\varepsilon_{0}>0$ such that

$$
\left(u_{0_{k}}, u_{1_{k}}\right) \rightarrow\left(\varphi_{\omega_{0}}, i \omega_{0} \varphi_{\omega_{0}}\right) \in X \oplus L_{r}^{2}
$$

and

$$
\inf _{\psi \in S_{\omega_{0}}} \varrho\left(u_{k}\left(t^{k}\right), \psi\right)+\left|u_{k t}\left(t^{k}\right)-i \omega_{0} \psi\right|_{2}>\varepsilon_{0} .
$$

From Lemma 3.2, $\exists$ subsequence also denote it by $\left(u_{k}\left(t^{k}\right)\right)$ such that

$$
d\left(\omega_{0}+1 / k\right) \leqq 1 / n \int\left|\nabla u_{k}\left(t^{k}\right)\right|^{2} d x \leqq d\left(\omega_{0}-1 / k\right),
$$

and $\left(\int\left|u_{k}\left(t^{k}\right)\right|^{2} d x\right)$ is bounded (by Theorem 1.1). Now as $k \rightarrow \infty$

$$
1 / n \int\left|\nabla u_{k}\left(t^{k}\right)\right|^{2} d x \rightarrow d\left(\omega_{0}\right)
$$

from continuity of $d(\omega)$. From Eq. (3.8) we have

$$
1 / 2 \int\left|v_{+k t}\left(t^{k}\right)\right|^{2} d x+J_{\omega_{+}}\left(u_{k}\left(t^{k}\right)\right)<d\left(\omega_{0}+1 / k\right),
$$

therefore $\exists$ subsequence such that

$$
J_{\omega_{0}}\left(u_{k}\left(t^{k}\right)\right) \rightarrow d^{1} \leqq d\left(\omega_{0}\right) .
$$

By Corollary 1.2 Eq. (3.9) and (3.10) imply that $\exists \psi \in S_{\omega_{0}}$ such that

$$
\begin{gathered}
\left\|u_{k}\left(t^{k}\right)-\psi\right\| \rightarrow 0, \\
J_{\omega_{0}}\left(u_{k}\left(t^{k}\right)\right) \rightarrow d\left(\omega_{0}\right) .
\end{gathered}
$$

Again from Eq. (3.8) we have

$$
\int\left|v_{+k t}\left(t^{k}\right)\right|^{2} d x=\left|u_{k t}\left(t^{k}\right)-i \omega_{+} u_{k}\left(t^{k}\right)\right|_{2}^{2} \rightarrow 0,
$$

and

$$
\left|\int\left(G\left(\left|u_{k}\left(t^{k}\right)\right|\right)-G(|\psi|)\right) d x\right| \rightarrow 0,
$$

which contradicts the assumption of instability.

\section{Examples}

We'll present here two examples where we have stable standing waves.

Theorem 4.1. The equation

$$
u_{t t}-\Delta u+u-|u|^{p-1} u=0, \quad x \in \mathbb{R}^{n}, \quad n \geqq 3,
$$

has stable standing waves for $1<p<1+4 / n$. 
Proof. In order to show the existence of stable standing waves it is sufficient to show that $d(\omega)$ is strictly convex for some interval of $\omega$.

Solution of the equation

$$
-\Delta \varphi_{\omega}+\left(1-\omega^{2}\right) \varphi_{\omega}-\left|\varphi_{\omega}\right|^{p-1} \varphi_{\omega}=0
$$

has the following scaling property: let $v(x)=(1 / \delta) \varphi_{\omega}(x / \beta)$, then

$$
-\delta \beta^{2} \Delta v+\left(1-\omega^{2}\right) \delta v-\delta^{p}|v|^{p-1} v=0,
$$

and for

$$
\beta^{2}=1-\omega^{2}=\delta^{p-1}
$$

Equation (4.1) becomes

$$
-\Delta v+v-|v|^{p-1} v=0 .
$$

Now

$$
\begin{aligned}
\int|\nabla v|^{2} d x= & \beta^{n-2} / \delta^{2} \int\left|\nabla \varphi_{\omega}\right|^{2} d x \\
& \Rightarrow \int\left|\nabla \varphi_{\omega}\right|^{2} d x=\left(1-\omega^{2}\right)^{\alpha} \int\left|\nabla \varphi_{0}\right|^{2} d x,
\end{aligned}
$$

where $\alpha=(4-(n-2)(p-1)) / 2(p-1)$.

Now it becomes easy to see when $d(\omega) \equiv 1 / n \int\left|\nabla \varphi_{\omega}\right|^{2} d x$ is strictly convex,

$$
\begin{gathered}
d(\omega)=\left(1-\omega^{2}\right)^{\alpha} / n \int\left|\nabla \varphi_{0}\right|^{2} d x=\left(1-\omega^{2}\right)^{\alpha} d(0), \\
\Rightarrow d^{\prime \prime}(\omega)=\left[-2 \alpha\left(1-\omega^{2}\right)^{\alpha-1}+4 \alpha(\alpha-1) \omega^{2}\left(1-\omega^{2}\right)^{\alpha-2}\right] d(0) \\
\Rightarrow d^{\prime \prime}(\omega)=2 \alpha\left[-1+(2 \alpha-1) \omega^{2}\right]\left(1-\omega^{2}\right)^{\alpha-2} d(0),
\end{gathered}
$$

since $1<p<1+4 / n, \alpha>0,2 \alpha-1>0$.

Therefore $d^{\prime \prime}(\omega)>0$ implies $-1+(2 \alpha-1) \omega^{2}>0$,

$$
\begin{gathered}
\omega^{2}>1 /(2 \alpha-1), \\
\alpha=(4-(n-2)(p-1)) / 2(p-1) .
\end{gathered}
$$

Moreover $\omega^{2}>1$ for Eq. (4.1) to have a solution. Therefore

$$
1>\omega^{2}>(p-1) /(4-(n-1)(p-1)),
$$

and this set is not empty for $1<p<1+4 / n$.

Remark 4.1. For $1+4 / n<p<1+4 /(n-2)$ we showed that all standing waves obtained by Theorem 1.1 are unstable [6].

Another example we'll consider is one which appears in studying spin-0 particles in field theory [4]. The potential, i.e. $G(|u|)$, for this model is of the form $G(|u|)=-|u|^{4} / 4+|u|^{6} / 6$.

Proposition 4.1. The equation

$$
-\Delta u+\left(1-\omega^{2}\right) u-|u|^{2} u+|u|^{4} u=0, \quad x \in \mathbb{R}^{3},
$$


has nontrivial solution $\varphi_{\omega}$ of lowest energy for $\omega^{2} \in(13 / 16,1)$. Moreover

$$
d(\omega) \equiv 1 / 3 \int\left|\nabla \varphi_{\omega}\right|^{2} d x \rightarrow \infty \quad \text { as } \quad \omega^{2} \rightarrow 13 / 16 .
$$

Proof. For Eq. (4.8) to have nontrivial solution it is sufficient to have $1-\omega^{2}>0$ and $\exists \eta$ such that $\left(1-\omega^{2}\right) \eta^{2} / 2+G(\eta)<0$. Now

$$
\left(1-\omega^{2}\right) \eta^{2} / 2-\eta^{4} / 4+\eta^{6} / 6<0
$$

for some $\eta$ if

$$
\begin{gathered}
(1 / 4)^{2}-4\left(1-\omega^{2}\right) / 12>0 \\
\Rightarrow \omega^{2}>13 / 16 .
\end{gathered}
$$

We show that $d(\omega) \rightarrow \infty$ as $\omega^{2} \rightarrow 13 / 16$ by contradiction. Assume that $d(\omega)$ remains bounded then by Theorem $1.1\left\|\varphi_{\omega}\right\|$ is bounded. This implies that $\exists$ sequence $\omega_{k} \rightarrow 13 / 16$ and $v \in H_{r}^{1}\left(\mathbb{R}^{3}\right)$ such that $\varphi_{\omega_{k}}{ }^{w} v \in H_{r}^{1}\left(\mathbb{R}^{3}\right)$. Again by Theorem 1.1

$$
K_{\omega_{0}}(v) \leqq \underline{\lim } K_{\omega_{0}}\left(\varphi_{\omega_{k}}\right)=\underline{\lim }\left(\omega_{k}^{2}-\omega_{0}^{2}\right) / 2 \int\left|\varphi_{\omega_{k}}\right|^{2} d x+K_{\omega_{k}}\left(\varphi_{\omega_{k}}\right),
$$

where $\omega_{0}^{2}=13 / 16$. But $K_{\omega_{k}}\left(\varphi_{\omega_{k}}\right)=0$, therefore

$$
K_{\omega_{0}}(v) \leqq 0 .
$$

Now $K_{\omega_{0}}(u)>0 \forall u \in H_{r}^{1}\left(R^{3}\right), u \neq 0$, so from (4.10) we have that $v=0$. By Eq. (4.9) we have that the convergence is strong. But $d(\omega)=1 / 2 \int\left|\nabla \varphi_{\omega}\right|^{2} d x$ is monotone decreasing function,

$$
d\left(\omega_{1}\right)>0 \Rightarrow 0=\varliminf_{k \rightarrow \infty} d\left(\omega_{k}\right)>d\left(\omega_{1}\right)>0
$$

a contradiction. Therefore $d(\omega) \rightarrow \infty$ as $\omega \rightarrow 13 / 16$.

Theorem 4.2. The equation

$$
u_{t t}-\Delta u+u-|u|^{2} u+|u|^{4} u=0, \quad x \in \mathbb{R}^{3}
$$

has stable standing waves for $\omega$ close to $13 / 16$.

Proof. By Proposition $4.1 d(\omega) \rightarrow \infty$ as $\omega \rightarrow 13 / 16$ and by Proposition $2.1 d(\omega)$ is monotone decreasing function of $\omega$. Therefore the graph of $d(\omega)$ looks like

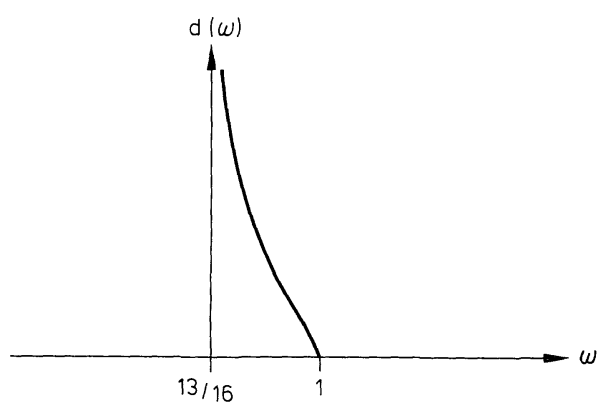

Fig. 4.1 
Now it is easy to see that $d(\omega)$ is strictly convex for $\omega$ close to $13 / 16$ and by Theorem 3.1 these standing waves are orbitally stable.

Remark 4.2. This particular example was studied numerically by Anderson [1] where he showed that for $\omega^{2}$ close to $13 / 16$ there are stable standing waves and that for $\omega$ close to 1 they are unstable and this is precisely what we show in [6]. Acknowledgement. I would like to thank Professor Walter Strauss for his helpful remarks.

\section{References}

1. Anderson, D.L.T.: J. Math. Phys. 12, 945-952 (1971)

2. Berestycki, H., Cazenave, T.: Instabilité des états stationnaires dans les équations de Schrödinger et de Klein-Gordon, non linéaires. C. R. Acad. Sci. 293A, 489-492 (1981)

3. Cazenave, T., Lions, P.L.: Orbital stability of standing waves for some nonlinear Schrödinger equations. Commun. Math. Phys. 85, 549-561 (1982)

4. Lee, T.D.: Particle physics and introduction to field theory. New York: Harwood Academic Publishers 1981

5. Lions, P.L.: Principle de concentration - compacite en calcul des variations. C. R. Acad. Sci. Paris 294, 261-264 (1982)

6. Shatah, J.M.: Unstable ground state and standing waves of nonlinear Klein-Gordon equations (to appear)

7. Strauss, W.A.: Nonlinear invariant wave equations. In: Lecture Notes in Physics, Vol. 23, pp. 197-249 (Erice 1977). Berlin, Heidelberg, New York: Springer 1978

8. Strauss, W.A.: Anais. Acad. Bras. Cienc. 42, 645-651 (1970)

9. Strauss, W.A.: Existence of solitary waves in higher dimensions. Commun. Math. Phys. 55, 149 (1977)

10. Berestycki, H., Lions, P.: Arch. Rat. Mech. Anal. (1983)

Communicated by A. Jaffe

Received November 8, 1982; in revised form May 11, 1983 
